\title{
Impact of genetic variations on pharmacokinetic and pharmacodynamic properties of medicines and the future of drug therapy
}

\begin{abstract}
Genetic variations between individuals usually leads to differences in the drug response as it could affect both pharmacokinetic and pharmacodynamic processes. An example of mutation of genes that affect drug targets (pharmacodynamic) is VKORC1 gene that codes for active sites of the enzyme vitamin $\mathrm{K}$ epoxide reductase that is responsible for activation vitamin $\mathrm{K}$. A famous example of mutation affecting pharmacodynamic properties of medicine is the anticancer agent irinotecan. Bone marrow suppression is a well known side effect for this medicine. In many cases, this adverse drug reaction appears to be very severe compared to the majority of the individuals, and that is possibly due to a mutation of a gene called UDP-GT 1A1.Identifying exact genes could be helpful in better targeting of medicines in the future, Pharmacogenetic testing and individualized medicine widens the window of therapy for better treatment approaches, it helps us to reduce both rate of toxicity and the dose of the drug, so it saves both lives and economically efficient that is why it worth funding by drug authorities in order for improving better and economically efficient clinical practice in the future
\end{abstract}

Keywords: Pharmacogenetic; Enzyme inhibitors; Individualized medicine; Mutation; Metabolizing enzymes.

Since the late 1950s, the effect of genetic makeup on drug response has been confirmed, and this has opened a wide window for discoveries concerning determining exact genes responsible for certain variations in drug response which in turn gave birth to a whole new medical field which is called individualized medicine. ${ }^{1}$ Scientists are working to divide humans into different populations based on their genetic makeup and determine drug doses depending on genetic background. Pharmacogenetic helps scientists in the process of individualizing medicine through pharmacogenetic testing of the individuals to see if they suit having certain medication or not, or to check the right dose for different individuals, lacking this mutation. ${ }^{2}$ For the time being, individualized medicine has a limited implementation, but it is considered to be very promising in the future of clinical practice as its only the beginning of this fields era. ${ }^{3}$ Genetic variations between individuals usually lead to differences in the drug response as it could affect both pharmacokinetic and pharmacodynamic processes, as genetic mutation could affect the gene that responsible for producing certain proteins that are involved in pharmacokinetics and pharmacodynamics of a certain medicine, and this could lead to triggering toxic reactions in the body as a result of enhancing its activity, or it could end up with reduction drug's activity that consequently leads to the failure of the therapy. ${ }^{4,5}$ Pharmacokinetic processes like absorption, distribution, metabolism, and excretion are affected because of the mutations that happen in genes that code for enzymes and transporters involved in these processes, like for example P-glycoprotein (which is an ATP dependent efflux pump) is expressed in many tissues

${ }^{1}$ Department of Pharmacology and Toxicology, College of Pharmacy, Hawler Medical University, Erbil, I raq.

* Correspondence: rojgar.hamed@hmu.edu.krd 
throughout the body like small intestine and blood brain barrier, and in some individuals who have a mutation in MDRB1 gene have higher expression of this protein, and this will lead to reduction in the absorption or penetration of drugs like HIV protease inhibitors, another example of mutations that changes drug response is in the CYP genes that codes for liver enzymes responsible for a phase II metabolism. ${ }^{6}$ For e.g CYP2D6 responsible for metabolism for a variety of drugs like antidepressants, patients carrying multiple copies of the active gene (UM) have higher rate of drug metabolism and this will lead to reduction in the response to class of drugs and failure of the treatment. ${ }^{7}$

Specific examples of the impact of genetic variation on pharmacodynamic properties

An example of mutation of genes that affect drug targets (pharmacodynamic) is VKORC1 gene that codes for active sites of the enzyme epoxide reductase that is responsible for activation vitamin $\mathrm{K}$ and further activation of coagulation factors. Warfarin, which is an inhibitor of the above enzyme, shows a reduced efficacy in individuals having a mutation at VKORC1 gene. Mutation in the mentioned gene increases the amount of the enzyme involved in vitamin $\mathrm{K}$ activation. As a result, a higher amount of vitamin $\mathrm{K}$ will be activated that needs a higher dose of warfarin to be inhibited. Thus, patients with this type of genetic mutation must have more than usual daily dose of warfarin 15 $\mathrm{mg}$ in order to develop response compared to patients lacking this mutation. ${ }^{8,9}$ Another famous example is the effect of genetic variants in the gene CHRNA7, which encodes for the major subunit of the acetylcholine receptor ( $\alpha 7-n A C h R)$, and is discovered to be associated with the clinical response to cholinesterase inhibitors (ChEl) in Alzheimer's disease (AD) patients. ${ }^{10}$ It has been shown that variant carriers using galantamine were 11 times more likely to be responders than female non-carriers using donepezil or rivastigmine. ${ }^{11}$

Specific examples of the impact of genetic variation on pharmacokinetic properties

Metabolism of drugs is a main pharmacokinetic process that many drugs passing through before they get excreted, metabolism is usually catalyzed by certain enzymes that are found in different parts of our body but mainly in the liver, the main enzyme involved in phase I metabolic processes is Cytochom P450, that has up to 21 families, ${ }^{12}$ and many other enzymes are involved in phase II metabolic processes like Glutathion-S-Transferase. ${ }^{13}$

A famous example of mutation affecting pharmacodynamic properties of medicine is the anticancer agent Irinotecan, ${ }^{14}$ Bone marrow suppression is a well known side effect for this medicine, but in many cases this adverse drug reaction appears to be very severe compared to the majority of the individuals, and that is possibly due to a mutation of a gene called UDP-GT $1 \mathrm{~A} 1$, this gene codes for an enzyme called UDP-Glucuronosyltransferase which is responsible for metabolism, hence deactivation, of Irinotecan, mutations in this gene could result in poor metabolizing ability of some individuals ending up with high concentration of the active drug that usually imparts myelosuppresion and severe diarrhea, ${ }^{15}$ that's why well respected organizations such as FDA has labeled Irinotecan for genotyping and not to be used without checking genetic map of this gene to see if the patient is eligible for dose reduction and this is in order to the possible adverse drug reaction that could happen in certain individuals. ${ }^{16}$ Another example is the role of CYP2D6 polymorphism in the efficacy and toxicity of Tramadol. This medicine is metabolized in the liver mainly through CYP2D6 enzyme into O-desmethyl-tramadol, which is four times more active than the parent drug. ${ }^{17,18}$ Studies have shown than an increased efficacy and toxicity has been shown in individuals with two active genes (homozygous) for CYP2D6 (extensive 
metabolizers) as toxic metabolites are excreted from the kidney. Studies showed that it might cause renal dysfunction in both healthy individuals and drug abusers. ${ }^{19,20}$ Another example of drug ineffectiveness due to extensive metabolism is the classic case of fast acetylators, plasma peak level for Isoniazide INH is one hour in fast acetylators while 3 hours in slow acetylators. ${ }^{21}$ Pharmacogenetic testing and individualized medicine widens the window of therapy for better treatment approaches, it helps us to reduce both rate of toxicity and the dose of the drug, so it saves both lives and economically efficient that is why it worth funding by drug authorities in order for improving better and economically efficient clinical practice in the future, ${ }^{22}$ and many systematic reviews identified robust evidence of the cost effectiveness of genotyping prior to treatment with a number of common drugs. ${ }^{23}$

\section{References}

1. Kalow W. Familial incidence of low pseudocholinesterase level. Lancet 1956; ii:5767.

2. Louca S. Personalized medicine-a tailored health care system, challenges and opportunities. Croat Med J 2012; 53:211-3.

3. Evans WE, Relling MV. Pharmacogenomics: translating functional genomics into rational therapies. Science 1999; 286(5439):487-91.

4. Weng L, Zhang L, Peng Y, Huang RS. Pharmacogenetics and pharmacogenomics: a bridge to individualized cancer therapy. Pharmacogenomics 2013; 14(3):315-24.

5. Lalovic B, Kharasch E, Hoffer C. Pharmacokinetics and pharmacodynamics of oral oxycodone in healthy human subjects: role of circulating active metabolites. Clin Pharmacol Ther 2006; 79(5):461-79.

6. Owen A, Chandler B, Back DJ. The implications of P-glycoprotein in HIV: friend or foe? Fundam Clin Pharmacol 2000; 19:283.

7. Kato M, Serretti A. Review and meta-analysis of antidepressant pharmacogenetic findings in major depressive disorder. Mol Psychiatry 2010; 15(5):473-50.

8. Rost S, Fregin A, Ivaskevicius V, Conzelmann E, Hortnagel K, Pelz $\mathrm{HJ}$, et al. Mutations in VKORC1 cause warfarin resistance and multiple coagulation factor deficiency type 2. Nature 2004; 427:537-41.
Mollnterv 2006; 6:223-7.

10. Barabash A, Marcos A, Ancin I, Vazquez-Alvarez $\mathrm{B}$, de Ugarte C. APOE, ACT and CHRNA7 genes in the conversion from amnestic mild cognitive impairment to Alzheimer's disease. Neurobiol Aging 2009; 30:1254-64.

11. Weng $P$, Chen J, Chen T, Sun Y, Wen L, Yip P. CHRNA7 Polymorphisms and Response to Cholinesterase Inhibitors in Alzheimer's disease. PLoS One 2013; 8(12):e84059.

12. Danielson PB. The cytochrome P450 superfamily: biochemistry, evolution and drug metabolism in humans. Current Drug Metabolism 2002; 3(6):561-97.

13. Allocati N, Masulli M, Di llio C, Federici L. Glutathione transferases: substrates, inihibitors and pro-drugs in cancer and neurodegenerative diseases. Oncogenesis 2018; 7(1):8.

14.Marsh S, Hoskins J. Irinotecan pharmacogenomics. Pharmacogenomics 2010; 11(7):1003-10.

15. Takano M, Sugiyama T. UGT1A1 polymorphisms in cancer: impact on irinotecan treatment. Pharmacogenomics and Personalized Medicine 2017; 10:61-8.

16. Schmoll HJ, Van Cutsem E, Stein A. ESMO Consensus Guidelines for management of patients with colon and rectal cancer. A personalized approach to clinical decision making. Ann Oncol 2012; 23(10):2479-16.

17. Bressolle F, Rochette A, Khier S, Dadure C, Ouaki J, Capdevila X. Population pharmacokinetics of the two enantiomers of tramadol and $\mathrm{O}$-demethyl tramadol after surgery in children. Br J Anaesth 2009; 102:390-9.

18. Zhou SF. Polymorphism of human cytochrome P450 2D6 and its clinical significance: Part II. Clin Pharmacokinet 2007; 48:761-804.

19. Stamer UM, MusshoffF, Kobilay M, Madea B, Hoeft A. Concentrations of tramadol and O-desmethyltramadol enantiomers in different CYP2D6 genotypes. Clin Pharmacol Ther 2007; 82:41-7.

20. Wang SQ, Li CS, Song YG. Multiply organ dysfunction syndrome due to tramadol intoxication alone. Am J Emerg Med 2009; 27:903.

21. Evans DA, Manley KA, Mckusick VA. Genetic control of isoniazid metabolism in man. $\mathrm{Br}$ Med $\mathrm{J}$ 1960; 2(5197):485-91.

22. Djalalov S, Musa Z, Mendelson M, Siminovitch $\mathrm{K}$, Hoch J. A review of economic evaluations of genetic testing services and interventions (2004-2009). Genet Med 2011; 3(2):89-94.

23. Plumpton CO, Roberts D, Pirmohamed $M$, Hughes DA. A systematic review of economic evaluations of pharmacogenetictesting for prevention of adverse drug reactions. Pharmacoeconomics 2016; 34(8):771-93. 